Pacific Journal of Mathematics

int 


\title{
ON THE NUMBER OF SINGULAR POINTS, LOCATED ON THE UNIT CIRCLE, OF CERTAIN FUNCTIONS REPRESENTED BY C-FRACTIONS
}

\author{
Vikramaditya Singh and W. J. ThroN
}

\section{The continued fraction}

$$
1+\stackrel{\mathrm{K}}{\mathrm{K}=1}_{\mathrm{K}}\left(\frac{d_{n} z^{\alpha} n}{1}\right)
$$

where the $\alpha_{n}$ are positive integers and the $d_{n} \neq 0$ for all $n \geqq 1$, are sometimes called $C$-fractions. They were first studied by Leighton and Scott [2]. It is well known (see [1]) that if

$$
\lim _{n \rightarrow \infty}\left|d_{n}\right|^{1 / \alpha_{n}}=1 \text { and } \lim _{n \rightarrow \infty} \alpha_{n}=\infty,
$$

the continued fraction (1) converges to a function which is meromorphic for all $|z|<1$. The first results concerning the location of singular points of functions of this type were obtained by Scott and Wall [4]. Considerably better results were recently obtained by one of the present authors $[5,6]$. In all of these results the continued fractions are assumed to satisfy the conditions (2) or even more restrictive ones. In this paper we are able to weaken condition (2) and replace it by

$$
\text { (a) } \quad \lim _{n \rightarrow \infty}\left(4\left|d_{n}\right|\right)^{1 / a_{n}=1} \text {, }
$$

$(2)^{\prime}$

(b) there exists a sequence $\left\{\alpha_{n_{k}}\right\}$ such that

$$
\lim _{k \rightarrow \infty} \alpha_{n_{k}}=\infty \text { and } \lim _{k \rightarrow \infty} n_{k} / k<2 .
$$

While all but one of the previous results gave sufficient conditions for the function represented by (1) to have the circle $|z|=1$ as a natural boundary, we give here criteria which are sufficient in order that the function has at least $p$ singular points on the circle.

Let $A_{n}(z) / B_{n}(z)$ be the $n$th approximant of (1) and let $\sigma_{n}$ and $\tau_{n}$ be the degrees of the polynomials $A_{n}(z)$ and $B_{n}(z)$, respectively. Also, let $\rho_{n}$ be the maximum of the degrees of $A_{n}^{*}(z)$ and $B_{n}^{*}(z)$ where $A_{n}^{*}(z) / B_{n}^{*}(z)$ is the $n$th approximant of (1) when the $d_{n}$ are replaced by their moduli. Then

Received April 1, 1954, and in revised form June 28, 1954. This research was supported by the United States Air Force, through the Office of Scientific Research of the Air Research and Dovelopment Command. 


$$
\sigma_{n} \leqq \rho_{n}, \quad \tau_{n} \leqq \rho_{n},
$$

Our theorem can now be stated as follows:

THEOREM. For the continued fraction (1) let $\rho_{n}$ be defined as above. Further let

$$
\begin{gathered}
h_{n}=\sum_{\nu=1}^{n+1} \alpha_{\nu}, \\
k=\liminf \frac{\rho_{n}}{h_{n}-\rho_{n}}, \\
\cos \Gamma= \begin{cases}1-\frac{2(k+\sqrt{ } k(1+2 k))}{1+2 k} & \text { if } 0<k \leqq 1 / 2, \\
-k, & \text { if } 1 / 2<k<1,\end{cases} \\
0 \leqq \Gamma<\pi, \\
p=[\pi / \Gamma]+1 .
\end{gathered}
$$

Here $[x]$ denotes the largest integer not exceeding $x$.

If the continued fraction satisfies conditions (2)' and if in addition $k<1$, then the function to which (1) converges for $|z|<1$ cannot be meromorphic on any arc of the unit circle of angular measurement greater than $2 \Gamma$. Thus the function has at least $p$ singular points, which are not poles, on the unit circle. If $k=0$ the function has the unit circle as a natural boundary.

2. To establish the theorem we first note that the condition (2)' (a) is sufficient to insure the convergence of (1) in the wider sense, and hence to a meromorphic function, for all $|z|<1$.

The condition (2)' (b) is sufficient to insure that

$$
\lim _{n \rightarrow \infty} \frac{h_{n}-\rho_{n}}{n+1}=\infty
$$

This is seen as follows. One shows by induction that $\rho_{n}$ is the sum of not more than $(n+1) / 2$ different $\alpha_{\nu}, \nu=1, \cdots, n$. Hence $h_{n}-\rho_{n}$ is the sum of at least $(n+1) / 2$ different $\alpha_{\nu}, \nu=1, \cdots, n+1$. Let $\left\{\alpha_{n_{k}}\right\}$ be the sequence which is assumed to satisfy $\lim _{k \rightarrow \infty} \alpha_{n_{k}}=\infty$. Now denote by $k(n)$ the number of elements of this subsequence which have a subscript less than or equal to $n$. Then $n_{k(n)}+1>n$. Also in view of the second condition in (2)' (b) $n_{k(n)+1}<(2-\varepsilon)(k(n)+1)$, for $n$ sufficiently large. Combining these two inequalities we obtain

$$
k(n)>n(1 / 2+\eta), \quad \text { for } n>n_{0} .
$$


It follows that $h_{n}-\rho_{n}$ contains at least $\eta n$ different $\alpha_{n_{k}}$, for all $n>n_{0}$. Now, if $M$ is a positive number, there exists an integer $k_{0}$ such that $\left|\alpha_{n_{k}}\right|>M$ for $k>k_{0}$. Then for all $n>\max \left(n_{0}, 2 k_{0} / \eta\right),\left(h_{n}-\rho_{n}\right) /(n+1)$ $>\eta n M / 2(n+1)$ and equation (5) is established.

Consider now the continued fraction

$$
1+\stackrel{K}{n=m}_{(}^{\infty}\left(\frac{d_{n} z^{\alpha} n}{1}\right)
$$

and denote by $h_{n}^{(m)}, \rho_{n}^{(m)}$ and $k^{(m)}$ the quantities in (6) corresponding to $h_{n}, \rho_{n}$ and $k$ in (1). It is then clear that the function $f_{m}(z)$ to which (6) converges and the function $f(z)$ to which (1) converges have, except for poles, the same singular points.

LEMMA 1.

$$
k^{(m)}=\liminf _{n \rightarrow \infty} \frac{\rho_{n}^{(m)}}{h_{n}^{(m)}-\rho_{n}^{(m)}}
$$

is independent of $m$.

Proof. We observe that ([3], p. 15)

$$
h_{n}=h_{m-2}+h_{n}^{(m)}
$$

and

$$
\rho_{n}^{(m)}+\rho_{m-3} \leqq \rho_{n} \leqq \rho_{n}^{(m)}+\rho_{m-1} .
$$

The lemma then follows if for a fixed $m$ we allow $n$ to tend to $\infty$.

It may also be noted that $\lim _{n \rightarrow \infty} \frac{h_{n}^{(m)}-\rho_{n}^{(m)}}{n-m+1}=\infty$, if (5) holds.

Let $\eta$ be an arbitrary positive quantity. There will then exist an $m$ such that

$$
\left|d_{n}\right|>\frac{1}{4}(1-\eta)^{\alpha_{n}} \quad \text { for } n>m
$$

The following lemma will be useful.

LEMMA 2. If $f(z)=\sum_{\nu=0}^{\infty} a_{2} z^{\nu}$ has a circle of convergence of radius greater than $\rho$ and if $h(z)=\sum_{\nu=0}^{\infty} b_{\nu} z^{\nu}$ is such that $h^{*}(\rho)=\sum_{\nu=0}^{\infty}\left|b_{\nu}\right| \rho^{\nu}$ exists and if $f(z) h(z)=\sum_{n=0}^{\infty} c_{n} z^{n}$ and $M=\max _{|z|=\rho}|f(z)|$, then

$$
\left|c_{n}\right|<\frac{M h^{*}(\rho)}{\rho^{n}} .
$$

Proof. To prove the lemma we note that by Cauchy's inequality 


$$
\left|a_{\nu}\right|<M / \rho^{\nu}
$$

Now $c_{n}=\sum_{\nu=0}^{n} a_{\nu} b_{n-\nu} ;$ hence,

$$
\begin{aligned}
\left|c_{n}\right| & \leqq \sum_{\nu=0}^{n}\left|a_{\nu}\right|\left|b_{n-\nu}\right| \leqq \sum_{\nu=0}^{n}\left|b_{n-\nu}\right| \frac{M}{\rho^{\nu}} \\
& =\frac{M}{\rho^{n}} \sum_{\nu=0}^{n}\left|b_{n-\nu}\right| \rho^{n-\nu}=\frac{M}{\rho^{n}} \sum_{\mu=0}^{n}\left|b_{\mu}\right| \rho^{\mu} \\
& \leqq \frac{M}{\rho^{n}} h^{*}(\rho) .
\end{aligned}
$$

This completes the proof of the lemma.

3. Now a meromorphic function $f(z)$ with $f(0)=1$ can be written as

$$
f(z)=\frac{p(z)}{q(z)},
$$

where $p(0)=q(0)=1$ and $p(z)$ and $q(z)$ are holomorphic wherever $f(z)$ is meromorphic. It is known (see [7]) that

$$
p(z) B_{n}(z)-q(z) A_{n}(z)=(-1)^{n}\left(\prod_{k=1}^{n+1} d_{k}\right) z^{n} n+\cdots .
$$

The omitted terms are of degree higher than $h_{n}$ in $z$. If we set

$$
\pi_{n}^{\prime}=\prod_{\substack{k=1 \\\left|a_{k}\right|>1}}^{n+1} d_{k}
$$

and introduce $A_{n}^{\prime}=A_{n}\left|\pi_{n}^{\prime}, B_{n}^{\prime}=B_{n}\right| \pi_{n}^{\prime}$, we have

$$
\begin{aligned}
& A_{n}^{\prime}(z)=\sum_{r=0}^{\sigma_{n}} a_{r}^{(n)} z^{r}, \\
& B_{n}^{\prime}(z)=\sum_{r=0}^{\tau_{n}} b_{r}^{(n)} z^{r} .
\end{aligned}
$$

It is easily seen (see [4]) that

$$
\left|a_{k}^{(n)}\right| \leqq 2^{n}, k \leqq \sigma_{n} ; \quad\left|b_{k}^{(n)}\right| \leqq 2^{n}, k \leqq \tau_{n} .
$$

We now introduce the transformation

$$
z=\varphi(w)=w \frac{1-\alpha w}{1-\beta w} \quad(0<\alpha<\beta<1),
$$


and note that $\varphi$ is holomorphic for $|w|<1 / \beta$ and that it reduces to the identity transformation as $\alpha \rightarrow \beta$. Replacing $z$ by $\varphi(w)$ in $f(z)$ we obtain

$$
F(w)=f(\varphi(w))=\frac{P(w)}{Q(w)}
$$

where $P(w)$ and $Q(w)$ may be so chosen that $P(0)=Q(0)=1$ and that $P$ and $Q$ are holomorphic wherever $F(w)$ is meromorphic. Relation (9) now becomes

$$
\begin{gathered}
P(w) B_{n}^{\prime}\left(w \frac{1-\alpha w}{1-\beta w}\right)-Q(w) A_{n}^{\prime}\left(w \frac{1-\alpha w}{1-\beta w}\right) \\
=(-1)^{n}\left(\prod_{\substack{k=1 \\
a_{k} \mid<1}}^{n+1} d_{k}\right) w^{h_{n}}+\cdots
\end{gathered}
$$

Let us set

$$
\begin{aligned}
& P(w)=1+p_{1} w+p_{2} w^{2}+\cdots, \\
& Q(w)=1+q_{1} w+q_{2} w^{2}+\cdots,
\end{aligned}
$$

and assume that both these series have a radius of convergence $\rho>1+\varepsilon$.

It is then easy to see that all ceofficients in the expansion, in powers of $(1+\varepsilon)$, of

$$
\left(\frac{1-\alpha(1+\varepsilon)}{1-\beta(1+\varepsilon)}\right)^{r}
$$

where $r$ is a positive integer, are positive. Hence if $\varepsilon$ is chosen so small that $1+\varepsilon<1 / \beta$, the lemma applied to the product

$$
P(w) B_{n}^{\prime}\left(w \frac{1-\alpha w}{1-\beta w}\right)=\sum_{m=0}^{\infty} c_{m} w^{m}
$$

yields

$$
\left|c_{m}\right| \leqq \frac{M_{1}}{(1+\varepsilon)^{m}} B_{n}^{\prime \prime}\left((1+\varepsilon) \frac{1-\alpha(1+\varepsilon)}{1-\beta(1+\varepsilon)}\right)
$$

Here $B_{n}^{\prime \prime}$ is the polynomial obtained from $B_{n}^{\prime}$ when each coefficient $b_{k}^{(n)}$ is replaced by $\left|b_{k}^{(n)}\right| . \quad M_{1}$ is the maximum of $|P(w)|$ for $|w|=1+\varepsilon$.

Similarly, for

$$
Q(w) A_{n}^{\prime}\left(w \frac{1-\alpha w}{1-\beta w}\right)=\sum_{m=0}^{\infty} d_{m} w^{m}
$$


one obtains

$$
\left|d_{m}\right| \leqq \frac{M_{2}}{(1+\varepsilon)^{m}} A_{n}^{\prime \prime}\left((1+\varepsilon) \frac{1-\alpha(1+\varepsilon)}{1-\beta(1+\varepsilon)}\right)
$$

Now set

$$
\Delta=\frac{1-\alpha(1+\varepsilon)}{1-\beta(1+\varepsilon)}
$$

With the help of (10) we obtain

$$
\max \left(A_{n}^{\prime \prime}((1+\varepsilon) \Delta), B_{n}^{\prime \prime}((1+\varepsilon) \Delta)\right) \leqq 2^{n} \frac{\{(1+\varepsilon) \Delta\}^{\rho_{n}+1}-1}{(1+\varepsilon) \Delta-1} .
$$

Thus if $M=\max \left(M_{1}, M_{2}\right)$ and $u_{h_{n}}$ is the coefficient of $w^{h_{n}}$ in the expansion of the left-hand side of (11), then

$$
\left|u_{h_{n}}\right| \leqq \frac{2^{n+1} M}{(1+\varepsilon)^{h_{n}}} \frac{\{(1+\varepsilon) \Delta\}^{\rho_{n}+1}}{(1+\varepsilon) \Delta-1} .
$$

If $u_{h_{n}^{(m)}}^{(m)}$ is the coefficient of $w_{n}^{h_{n}^{(m)}}$ in the expansion of the left-hand side of (11) for the continued fraction (6) we have, in complete analogy to $(14)$,

$$
\left|u_{h_{n}^{(m)}}^{(m)}\right| \leqq \frac{2^{n-m+2} M^{\prime}}{(1+\varepsilon)^{h_{n}^{(m)}}} \frac{\{(1+\varepsilon) \Delta\}^{\rho_{n}^{(m)}+1}}{(1+\varepsilon) \Delta-1} .
$$

On the other hand, for $m$ large enough, one can write

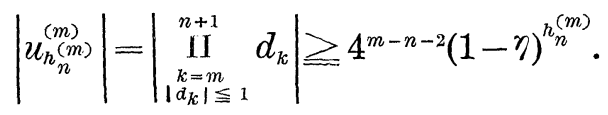

Thus,

$$
(1-\eta)^{h_{n}^{(m)}} \leqq 8^{n-m+2} \frac{M^{\prime}}{(1+\varepsilon)^{h_{n}^{(m)}-\rho_{n}^{(m)}-1}} \frac{\Delta^{\rho_{n}^{(m)}+1}}{(1+\varepsilon) \Delta-1} .
$$

Taking the $\left(h_{n}^{(m)}-\rho_{n}^{(m)}\right)$ root on both sides and letting $n$ tend to infinity we obtain

$$
(1-\eta)^{1+k^{(m)}} \leqq \frac{\Delta^{k^{(m)}}}{1+\varepsilon}
$$

Here $k^{(m)}$ is independent of $m$. The same applies to $\varepsilon$ since it depends on those singularities of $f_{m}(z)$ which are not poles and these, as we pointed out earlier, are the same as those of $f(z)$. Thus we can omit 
the $m$ and can let $\eta$ tend to zero, since it can be arbitrarily small, and obtain

$$
1 \leqq \frac{\Delta^{k}}{1+\varepsilon}
$$

This can be rewritten as

$$
(1+\varepsilon)^{1 / k} \leqq \frac{1-\alpha(1+\varepsilon)}{1-\beta(1+\varepsilon)}
$$

Now making use of the fact that $1+\varepsilon / k \leqq(1+\varepsilon)^{1 / k}$ for $k \leqq 1$ we can write

$$
1+\varepsilon / k \leqq 1+\frac{(\beta-\alpha)(1+\varepsilon)}{1-\beta(1+\varepsilon)}
$$

Here we set $s \geqq 1 /(1-\beta(1+\varepsilon))$ and solve for $\varepsilon$. Since $s>1 /(1-\beta)$ this leads to

$$
\varepsilon \leqq \frac{\beta-\alpha}{(1-\beta)} \frac{k}{1-k s(\beta-\alpha)}
$$

Thus by imposing on $\varepsilon$ the condition

$$
\varepsilon<\frac{s-1}{s} \cdot \frac{1}{\beta}-1, \quad s>1,
$$

which insures a fortiori that $1+\varepsilon<1 / \beta$, we have obtained in (15) a further condition which $\varepsilon$ must satisfy. It is clear that for $\alpha$ sufficiently close to $\beta$ this second condition becomes much stronger than the one arbitrarily imposed on $\varepsilon$. This allows us to conclude that in a circle of radius $1+\varepsilon^{\prime}$, where $\varepsilon^{\prime}$ fails to satisfy (15), at least one of the two functions $P$ or $Q$ must have a singular point and hence $F(w)$ must have a singular point not a pole somewhere in $|w|<1+\varepsilon^{\prime}$. Since $\varphi(w)$ has no singular points in $|w| \leqq 1+\varepsilon^{\prime}<1 \mid \beta$, the singular points of $F(w)$ in this region are caused by singular points of $f(z)$ in the image of this region under the mapping $z=\varphi(w)$. Since $f(z)$ is known not to have any singular points in $|z|<1$ we can further narrow down the location of the singular points to that part of the image of $|w| \leqq 1+\varepsilon^{\prime}$ which is outside the unit circle in the $z$-plane. This is a sickle-shaped region which as $\alpha \rightarrow \beta$ contracts to an arc of the unit circle symmetric about $z=1$. Our aim now is to choose $\beta$ ( $k$ of course is fixed) such that this arc becomes as small as possible.

Unless $\varepsilon<(\beta-\alpha)(1+\varepsilon) /(1+\beta(1+\varepsilon))$, the arc discussed above will be all of the unit circle. This suggests that $\beta$ should be so chosen that 


$$
k<\frac{1-\beta}{1+\beta+s(\beta-\alpha)(1-\beta)} .
$$

Since we are interested only in the case where $\beta-\alpha$ is very small, we take

$$
k=\theta \frac{1-\beta}{1+\beta}, \quad \theta<1
$$

that is

$$
\beta=\frac{1-k / \theta}{1+k / \theta}
$$

Clearly this can lead to a $\beta>0$ only if $k<\theta<1$. Substituting (16) in (15) we obtain

$$
\varepsilon=\frac{(\beta-\alpha)}{1+\beta} \theta^{\prime}, \quad \theta^{\prime}=\theta+O(\beta-\alpha) .
$$

Let $\gamma$ be the angle at which the circle $|z|=1$ is cut by the image of the circle $|w|=1+\varepsilon$ under the transformation $\varphi$. If $w_{0}$ is such that $\left|\varphi\left(w_{0}\right)\right|=1$ then $\gamma=\arg w_{0}+O(\beta-\alpha)$ and, hence,

$$
2 \cos \gamma=(1+\varepsilon)(\beta+\alpha(1+\varepsilon))-\frac{\varepsilon(2+\varepsilon)}{(1+\varepsilon)\left(\beta-\alpha(1+\varepsilon)^{2}\right)}+O(\beta-\alpha) .
$$

From (18) follows

$$
\beta-\alpha(1+\varepsilon)^{2}=(\beta-\alpha)\left(1-\frac{\alpha \theta^{\prime}}{1+\beta}(2+\varepsilon)\right),
$$

and hence

$$
\cos \gamma=\beta-\frac{\theta^{\prime}}{\left(1+\beta-2 \beta \theta^{\prime}\right)}+O(\beta-\alpha) .
$$

Noting that as $\alpha \rightarrow \beta, \theta^{\prime} \rightarrow \theta$ we can write

$$
\cos \gamma=\beta-\frac{\theta}{(1+\beta-2 \beta \theta)}+O(\beta-\alpha) .
$$

Thus if we denote by $\gamma^{*}$ the limiting value of $\gamma$ as $\alpha \rightarrow \beta$ and replace $\beta$ by its value in terms of $k$ we have

$$
\cos \gamma^{*}=1-\frac{4 k(1+k)+(\theta-k)^{2}}{2(\theta+k)(1+k-\theta)} .
$$

The minimum value of $\gamma^{*}$, say $\Gamma_{1}$ is attained for $0<k<1 / 2$ for 


$$
\theta=\frac{-k(5+6 k)+2(2 k+1) \sqrt{k(2 k+1)}}{1-2 k} .
$$

For $k=1 / 2$, we have $\theta=1 / 2$ and for $k>1 / 2$, since $\theta$ is restricted to the range $k<\theta<1$, we find that $\gamma^{*}$ is minimized for $\theta=k$. The resulting values are

$$
\cos \Gamma= \begin{cases}1-\frac{2}{1+2 k}(k+\sqrt{k(1+2 k)}), & \text { if } 0<k \leqq 1 / 2, \\ -k & , \quad \text { if } 1 / 2<k<1,\end{cases}
$$

To complete the proof of the theorem it now suffices to observe that the conditions imposed on $f(z)$ hold equally well when $z$ is replaced by $z e^{i \psi}$ for arbitrary $\psi$. It follows that there is no arc of angular measurement larger than $2 \Gamma$ on the circle $|z|=1$ which is free from singular points. From this follows immediately that the minimum number of singular points of the function is $[\pi / \Gamma]+1$.

We conclude by observing that if there exists a subsequence $\left\{\alpha_{n_{m}}\right\}$ such that

$$
\lim _{m \rightarrow \infty} \frac{\alpha_{n_{m}}}{h_{n_{m}-2}}=A>1
$$

then $\quad \lim _{m \rightarrow \infty} \frac{\rho_{n_{m}-1}}{h_{n_{m}-1}-\rho_{n_{m}-1}} \leqq \lim _{m \rightarrow \infty} \frac{h_{n_{m}-2}}{\alpha_{n_{m}}}=1 / A$.

It follows that $k \leqq 1 / A<1$ and we can state the following corollary to our main theorem.

COROLLARY. If the continued fraction (1) satisfies conditions (2)' and (19) then the conclusions of the theorem are valid with $k \leqq 1 / A$.

\section{REFERENCES}

1. W. Leighton, A test ratio for continued fractions, Bull. Amer. Math. Soc., 45 (1939) 97-100.

2. W. Leighton and W. T. Scott, A general continued fraction expansion, Bull. Amer. Math. Soc., 45 (1939), 596-605.

3. O. Perron, Die Lehre von den Kettenbruchen, Leipzig, 1929.

4. W. T. Scott and H. S. Wall, Continued fraction expansions for arbitrary power series, Ann. of Math., (2), 41 (1940), 328-349.

5. W. J. Thron, Singular points of functions defined by C-fractions, Proc. Nat. Acad. Sci. U.S.A., 36 (1950), 51-54.

6. - A class of meromorphic functions having the unit circle as a natural boundary, Duke Math. J., 20 (1953), 195-198.

7. H. S. Wall, Analytic theory of continued fractions, New York, 1948. 



\section{PACIFIC JOURNAL OF MATHEMATICS}

\section{EDITORS}

H. L. Royden

Stanford University

Stanford, California

E. HewitT

University of Washington

Seattle 5 , Washington
R. P. Dilworth

California Institute of Technology Pasadena 4, California

E. G. Straus

University of California

Los Angeles 24, California

\section{ASSOCIATE EDITORS}

E. F. BECKENBACH

C. E. BURGESS

H. BUSEMANN

H. FEDERER

\author{
M. HALL \\ P. R. HALMOS \\ V. GANAPATHY IYER \\ R. D. JAMES
}

M. S. KNEBELMAN

I. NIVEN

T. G. OSTROM

M. M. SCHIFFER
J. J. STOKER

G. SZEKERES

F. WOLF

K. YOSIDA

\section{SUPPORTING INSTITUTIONS}

UNIVERSITY OF BRITISH COLUMBIA

CALIFORNIA INSTITUTE OF TECHNOLOGY

UNIVERSITY OF CALIFORNIA

MONTANA STATE UNIVERSITY

UNIVERSITY OF NEVADA

OREGON STATE COLLEGE

UNIVERSITY OF OREGON

UNIVERSITY OF SOUTHERN CALIFORNIA
STANFORD UNIVERSITY

UNIVERSITY OF UTAH

WASHINGTON STATE COLLEGE

UNIVERSITY OF WASHINGTON

AMERICAN MATHEMATICAL SOCIETY CALIFORNIA RESEARCH CORPORATION HUGHES AIRCRAFT COMPANY 


\section{Pacific Journal of Mathematics}

\section{Vol. 6, No. $1 \quad$ November, 1956}

David Blackwell, An analog of the minimax theorem for vector payoffs..... 1

L. W. Cohen, A non-archimedian measure in the space of real

sequences ..................................... 9

George Bernard Dantzig, Constructive proof of the Min-Max theorem ..... 25

Jim Douglas, On the numerical integration of quasilinear parabolic

differential equations ............................... 35

James Michael Gardner Fell, A note on abstract measure ............. 43

Isidore Isaac Hirschman, Jr., A note on orthogonal systems . . . . . . . . . . 47

Frank Harary, On the number of dissimilar line-subgraphs of a given

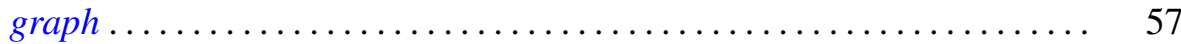

Newton Seymour Hawley, Complex bundles with Abelian group......... 65

Alan Jerome Hoffman, Morris Newman, Ernst Gabor Straus and Olga

Taussky, On the number of absolute points of a correlation ...........

Ernst Gabor Straus and Olga Taussky, Remark on the preceding paper.

Algebraic equations satisfied by roots of natural numbers . . ........ 97

Ralph D. James, Summable trigonometric series ................. 99

Gerald R. Mac Lane, Limits of rational functions . . . . . . . . . . . . . . . 111

F. Oberhettinger, Note on the Lerch zeta function ................. 117

Gerald C. Preston, On locally compact totally disconnected Abelian groups and their character groups ........................... 121

Vikramaditya Singh and W. J. Thron, On the number of singular points, located on the unit circle, of certain functions represented by

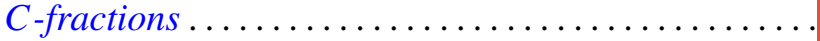

Sherman K. Stein, The symmetry function in a convex body ... 145 Edwin Weiss, Boundedness in topological rings.............

Albert Leon Whiteman, A sum connected with the series for the partition

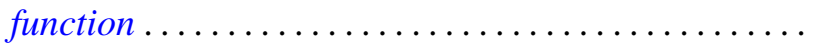

Alfred B. Willcox, Some structure theorems for a class of Banach algebras.

Joseph Lawrence Zemmer, Some remarks on p-rings and their Boolean geometry... 